\title{
Regeneration of the peripheral nerve - Development and evaluation of guide tubes of biodegradable polymer
}

\author{
Joana Gomes, José Domingos Santos, Ana Colette Maurício
}

\begin{abstract}
Damage to peripheral nerve fibers results in axonal loss and demyelination followed by regeneration and remyelination under optimal conditions with the possibility of some functional recovery. The experimental challenge is to accelerate axonal regeneration to promote reinnervation and improve functional recovery after peripheral nerve injury. In the past few decades, different types of biological or artificial guide tubes have been developed to bridge the gap of a sectioned nerve, to limit the fibrosis process and to orient the regenerating fibers towards the distal stump. Chitosan is widely used for biomedical applications, including crosslinked with other materials. In this work, chitosan guide tubes were produced and implanted using the rat sciatic nerve animal model. Functional tests were performed as well as a mechanical and structural characterization of the guide tubes.
\end{abstract}

\section{INTRODUCTION}

It is estimated that about $2.8 \%$ of trauma patients, many of whom have acquired life-long disabilities, are affected by peripheral nerve injuries. Unlike what happens in the central nervous system of mammals, the peripheral nervous system has a permissive environment provided by the Schwann cells[1][2]. Peripheral nerve injuries may include or not gaps between the nerve stumps. If an intervention is not made after an injury occurs, functional regeneration becomes restricted due to scars, neuroma formation or mismatched fibers[3]. In neurotmesis injuries, the nerve is completely transected. Without a surgical reconstruction the axonal regrowth is impossible, making this the most challenging type of peripheral nerve injury repair[4]. The tissue engineering approach is to repair, complement and regenerate damaged tissue by using materials that will support and reinforce the regenerating tissue. One of the strategies is to build threedimensional, porous scaffolds which role is to manipulate cell functions since they provide spaces and surface area enough for cell adhesion and proliferation, and to supply oxygen and nourishment. These scaffolds should be biocompatible and biodegradable, have a large surface to volume ratio, and be mechanically strong and capable of being formed into desired shapes and structures[5].

Joana Gomes is with Faculty of Engineering of Porto University, Porto, 4200-465 Portugal (corresponding author to provide e-mail: joanamsgomes@gmail.com).

José Domingos Santos, is with Faculty of Engineering of Porto University, Porto, 4200-465 Portugal (e-mail: jdsantos@fe.up.pt).

Ana Colette Maurício is with Institute of Biomedical Sciences of Abel Salazar, University of Porto, Porto, 4050-313 Portugal (e-mail: ana.colette@hotmail.com).

\section{CHITOSAN}

Chitosan is a natural and hydrophilic copolymer of Dglucosamine and $\mathrm{N}$-acetylglucosamine units and it is obtained from full or partial $\mathrm{N}$-deacetylation of chitin. Its biodegradability, biocompatibility, nonantigenicity, promotion of cell adhesion and nontoxicity has made it possible to use chitosan in a number of biomedical applications[6]. Being fragile in the dried form, chitosan can be chemically crosslinked or jointly used with other materials before scaffold fabrication. Chitosan matrices have low mechanical strength under physiological conditions and are unable to maintain a predefined shape after transplantation[7]. Their mechanical properties can be improved by adding $\gamma$-glycidoxypropyltrimethoxysilane (GPTMS) as a crosslinker, which is a silane coupling-agent with epoxy and methoxysilane groups. This modification, along with a freeze-drying technique, has been shown to produce chitosan type III membranes with $90 \%$ porosity and successful improvement of rat sciatic nerve regeneration after axonotmesis. It was concluded that this material, apart from working as a simple mechanical scaffold, could also work as an inducer of nerve regeneration[8][9].

The goals established for this work were to prepare chitosan guide tubes crosslinked with GPTMS, to mechanically and structurally characterize them, and finally to implant them in a group of animals. This would allow to compare the obtained results with others already described and published and to assess if improvements were achieved.

\section{MATERIALS AND METHODS}

Both chitosan (degree of deacetylation $>75 \%$; SigmaAldrich $^{\circledR}$, USA) and GPTMS (Sigma-Aldrich ${ }^{\circledR}$, USA) were dissolved separately in a $0.25 \mathrm{M}$ acetic acid solution in order to attain a concentration of $2 \%(\mathrm{w} / \mathrm{v})$ and $27.7 \%(\mathrm{w} / \mathrm{v})$, respectively. For the crosslinking to occur, the GPTMS solution was added to the chitosan solution to obtain a molar ratio of 1:0.5 (chitosan:GPTMS). The resultant chitosanGPTMS solution was poured into teflon molds, frozen at $20^{\circ} \mathrm{C}$ and then lyophilized to complete dryness. The resultant guide tubes were soaked in a $0.1 \mathrm{M} \mathrm{NaOH}$ aqueous solution to neutralize remaining residues of acetic acid and then lyophilized once again. The morphology and microstructure of the chitosan-GPTMS guide tubes were analyzed under a scanning electron microscope (SEM) with an accelerating voltage of $5 \mathrm{kV}$. The guide tubes and chitosan powder were characterized by Fourier Transform Infrared (FTIR) spectroscopy. Sixty-four scans were performed with a resolution of $4 \mathrm{~cm}^{-1}$. To evaluate the water uptake of the guide tubes, the samples were soaked in a phosphate-buffered 
saline (PBS) solution at $\mathrm{pH} 7.4$ at $37^{\circ} \mathrm{C}$ during 7 days. The water uptake was calculated using Equation (1) where $\mathrm{W}_{\mathrm{w}}$ is the weight of the wet guide tubes and $\mathrm{W}_{\mathrm{d}}$ is the weight of the dry guide tubes. The degree of crosslinking (DC) of the guide tubes was determined by the ninhydrin (NHN) assay, which determines the percentage of free amino groups $\left(\mathrm{NH}_{2}\right)$ remaining in the guide tubes after crosslinking, according to a method previously described in the literature[10]. The mechanical behavior of the guide tubes was evaluated and stress-strain curves were obtained for both dry and hydrated samples. Strip-shaped membranes were stretched using a crosshead speed of $1 \mathrm{~mm} / \mathrm{min}$. Young's modulus, i.e. the elastic modulus, was calculated from the stress at break and the strain at break.

In vivo testing was performed in four adult male Saco Sprague Dawley rats on which was inflicted a neurotmesis injury in the right sciatic nerve surgically reconstructed with a crosslinked chitosan guide tube. For the functional assessment, all animals were tested pre-operatively (week 0), at week 1 and every two weeks after the surgery, for a total period of follow-up of 20 weeks. ${ }^{1}$ Motor performance and nociceptive function were evaluated by measuring extensor postural thrust (EPT) and withdrawal reflex latency (WRL), respectively. In the first test, the force applied by the rat in grams was recorded, for both the healthy and affected hindlimb. The normal EPT (NEPT) (regarding the healthy hindlimb) and the experimental EPT (EEPT) values were used in Equation (3) to derive the percentage of functional deficit. The second test, called the hotplate test, consisted of placing the healthy hind paw of the rat in a plate at $56^{\circ} \mathrm{C}$ and measuring the time elapsed until its withdrawal. The same was performed for the affected hind paw. If there was no hind paw withdrawal after 12 seconds, the heat stimulus would be ceased in order to prevent tissue damage. For sciatic functional index (SFI), the animals were tested in a confined walkway where they were put to walk in order to get their footprints on paper. Some measurements were taken such as the distance from the heel to the third toe (print length)(PL); the distance from the first to the fifth toe (toe spread)(TS); and the distance from the second to the fourth toe (intermediary toe spread)(ITS). For both dynamic (SFI) and static assessment (SSI) all measurements were taken from the experimental (E) and normal (N) hindlimbs. Concerning SFI and SSI, an index of 0 is considered normal and an index of 100 indicates total impairment. All the results obtained for this group of four animals were then compared to those of other experimental groups. In control group none of the animals had a sciatic nerve injury; in gap group the injured nerve was left without any repair intervention; in end-to-end group immediate coaptation with epineurial sutures of the two transected nerve stumps was performed; in graft group it was obtained a nerve graft from the animal itself which was inverted $180^{\circ}$ and then sutured; in PLGA group a poly(Llactic-co-glycolic acid) (PLGA) guide tube was sutured to a transected sciatic nerve. Opposite leg and sciatic nerve were left intact in all groups and served as control for normal nerves.

\footnotetext{
${ }^{1}$ At the time of the thesis submission, it was only possible to perform the functional tests up until the fourth week.
}

\section{RESULtS}

Fig. 1 shows the microstructure of the chitosan-GPTMS guide tubes, which are highly porous. This is in accordance with previous studies where investigators obtained chitosanGPTMS membranes with a porosity of $90 \%$ and $110 \mu \mathrm{m}$ pores[5].

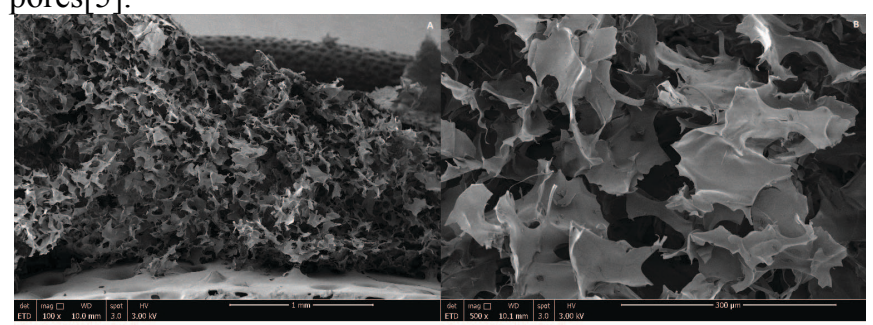

Figure 1. SEM images of the chitosan-GPTMS guide tubes.

In the FTIR spectrum of chitosan (Fig. 2) the bands around $1650 \mathrm{~cm}^{-1}$ and $1565 \mathrm{~cm}^{-1}$ are attributed to the amine bands I and II, respectively[11]. The reaction between amine groups present in chitosan and oxirane groups in GPTMS can be located in the transmittance band at $1550 \mathrm{~cm}^{-1}$.

The water uptake of the chitosan-GPTMS guide tubes was evaluated during 7 days. In the first two hours, they quickly absorbed the fluids being the water uptake nearly $1000 \%$ of the initial dry mass. However, after 24 hours this value started to decreased which may be due to mass loss.

As mentioned before, GPTMS was used as a crosslinker in the preparation of the guide tubes. Their degree of crosslinking was around $69 \%$.

Stress-strain curves were plotted and Young's modulus (E) was calculated for both dry and hydrated samples at a fixed strain of $2 \%$. It was observed an increase in the stress with increasing strain until material collapse occurred. Hydrated samples presented ultimate tensile stress lower than that of dry samples $(0.02 \pm 0.02$ and $0.18 \pm 0.08 \mathrm{MPa}$, respectively) due to the amount of water that filled the porous structure. Water might also work as a plasticizer agent, leading to a decrease of the Young's modulus from $2.91 \pm 2.20 \mathrm{MPa}$ for the dry samples to $0.19 \pm 0.05 \mathrm{MPa}$ for the hydrated samples.

FTIR (Ch-GPTMS)
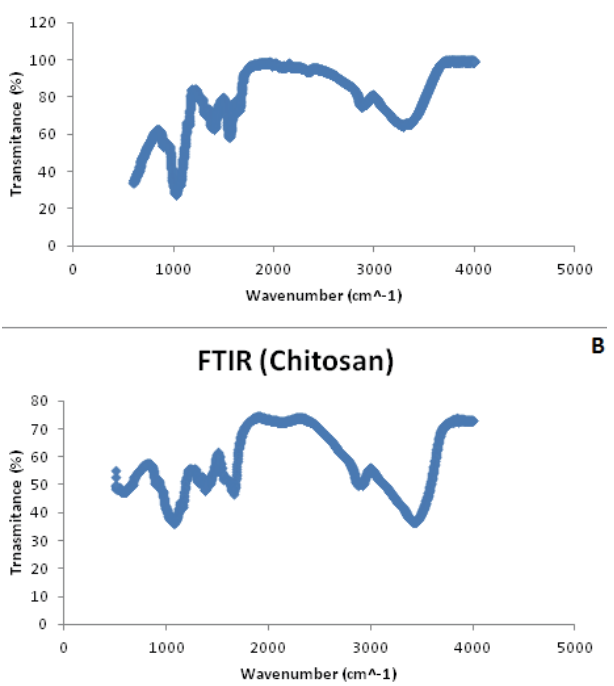

Figure 2. FTIR spectrum of chitosan-GPTMS (A) and chitosan powder (B). 
The results of the motor deficit (Fig. 3) showed that nerve transection (neurotmesis) caused a severe motor deficit in all experimental groups except in control group. One week after the intervention, the five groups severe loss of hindlimb extension force. During the follow-up period of 20 weeks, it can be observed that the motor deficit decreases, with a faster recovery along time in some groups than in others. However, in the chitosan-GPTMS (Ch-GPTMS) group there is no evidence of motor deficit decrease since the results concerning this group only go as far as week 4 post-surgery.

Fig. 3 also shows the result for the WRL test. In the first week post-surgery, all animals presented severe loss of sensory function and all tests had to be interrupted at the selected cutoff time of 12 seconds except for control group. Throughout the follow-up time the nociception function was recovered and at week 20 it was in the range of normal values (bellow 4.3 seconds) except for gap group, which did not present any recovery of the WRL, since the neurotmesis injury was not surgically repaired. The Ch-GPTMS group was already showing some decrease of the WRL at week 4 . At the end of the 20 weeks, the results of the hotplate test were similar in all four experimental treated groups. Gap group showed no signs of recovery in sensory function.

Both SFI and SSI were evaluated only in a small number of animals and not for all the experimental groups. At week 0 (pre-operative) SFI was $-7.4 \pm 5.4$ in the gap group. One week after surgeries, SFI was $-83.2 \pm 2.2$ for graft group. This results indicates a profound impairment in foot and toes ground contact. By the end of the follow-up period, SFI values remained highly negative which indicated only a modest recovery of the foot placement after neurotmesis. At week 20, the PLGA group had a SFI value of $-62.3 \pm 2.3$ and for the gap group -82.5 \pm 4.3 [12]. Variations on the SSI results were similar to SFI's. By week 20, SSI values were $-76.6 \pm 4.9$ for the gap group. For the Ch-GPTMS group SFI was $55.2 \pm 0.0$ in both week 1 and week 2 post-operative. At week 4, SFI became even more negative which translates the great impairment in foot and toes ground contact. It was verified in almost experimental rats autotomy, which limits the SFI test evaluation.

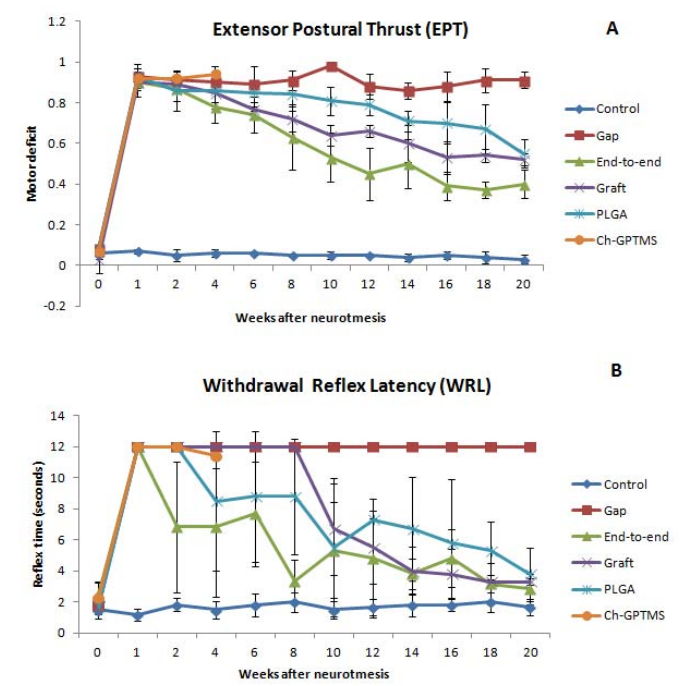

Figure 3. EPT and WRL results for 20 weeks follow-up.

\section{DISCUSSION}

In the present study, chitosan-GPTMS guide tubes were produced and a number of assays were performed in order to characterize them mechanically and structurally. Also, in vivo testing took place using the rat sciatic nerve as the experimental model. Peripheral nerve regeneration was assessed after a neurotmesis injury was induced.

The FTIR spectrum provides information through band properties, frequencies and intensities and it can be used to predict chemical processes and identify species[11]. The $2840 \mathrm{~cm}^{-1}$ peak is assigned to the methoxy group of the GPTMS and the 1192 and $914 \mathrm{~cm}^{-1}$ peaks are assigned to the epoxy group, which shows the presence of the crosslinker in the guide tubes[13].

The ninhydrin reaction determined quantitatively the amount of free amino groups, thus calculating the DC of the chitosan-GPTMS guide tubes. Of all the cyclic units of chitosan, $79 \%$ of them has an amino group that force-opens the epoxy group of GPTMS to form a -NH-O- bond[5]. During the crosslinking reaction, the epoxy group is linked to the amino group of chitosan and the methoxysilane groups are hydrolyzed forming silanol groups. These are subjected to the construction of a siloxane network due to condensation. Furthermore, the negative charges of the silanol groups and the positive charges of the amino groups favor the crosslinking reaction[13].

Observing Fig. 1, it becomes clear that the chitosanGPTMS guide tubes are highly porous, which in some extent is due to the presence of GPTMS that has been shown to be responsible for the size of the pores. Also, the pore size is strongly dependent on the freezing temperature, being that the size increased with the freezing temperature[5]. The guide tubes were developed in order to be porous while preserving some mechanical strength, which is a factor to be considered in order to avoid problems during transplantation and healing period such as collapsing and disintegration. The balance between hardness and flexibility should also be taken into account[14]. Concerning the mechanical test, the hydrated samples had a lower Young's modulus, which means that when in an environment with fluids, the guide tubes are less rigid and brittle. Thus, they become more prone to collapse than when dry.

During the period of swelling, the porous structures were filled with PBS and can go through a degradation process. At this point, the water occupies the atomic level space in the matrix as well as the pores. This demonstrates the ability of the matrix of the guide tubes to hold water, which is higher than the ability of the original chitosan[8]. These observations can be correlated with the SEM images, confirming the high values of water uptake.

The rat sciatic nerve has been the most commonly used animal model for studies concerning peripheral nerve regeneration due to its reliability[15]. Both functional and morphological assessments are evaluation tools when it is important to quantify the regeneration of the peripheral nerve considering different therapeutic strategies. ChitosanGPTMS structures have shown in in vivo studies an improved 
nerve fiber regeneration and functional recovery in neurotmesis injuries. The EPT and WRL tests have been proven to be reliable, valid and highly efficient tests when it comes to determine the functional recovery following sciatic nerve injury. They also provide a quantitative measurement of motor and sensitive function recoveries, respectively, and give consistently less variation between measurements. SFI tests provide a quantitative measure of degree of functional deficit, but can be cumbersome, time consuming and variable, especially considering the evaluation of neurotmesis injuries recoveries[16].

The surgeries allowed evaluating the performance of the chitosan-GPTMS guide tubes when in contact with fluids. The main observation was their friability which in some way is consistent with what was previously mentioned about the porosity, water uptake and mechanical strength. However, their mechanical behavior can be improved if an optimal ratio is achieved between chitosan and the crosslinker.

\section{CONCLUSION}

The goals initially proposed for this experimental work were accomplished. From the mechanical and structural analysis, the chitosan-GPTMS guide tubes seem to be suitable and in some way ideal to promote the nerve regeneration after a neurotmesis injury. However, the process of preparation of the guide tubes has yet alterations and improvements to be made in order to reach optimal conditions and better results.

$$
\begin{gathered}
\text { APPENDIX } \\
\text { Water uptake }(\%)=\frac{W_{w}-W_{d}}{W_{d}} \times 100 \\
\% D C=\frac{N_{N H r} a_{\text {fresh }}-N H N r a_{\text {fixed }}}{N H N r a_{\text {fresh }}} \times 100 \\
\% \text { Motor deficit }=\frac{N E P T-E E P T}{N E P T} \times 100 \\
\text { Toe spread factor }(\text { TSF })=\frac{E T S-N T S}{N T S} \\
\text { Intermediate toe spread factor }(I T S F)=\frac{E I T S-N I T S}{N I T S} \\
\text { Print length factor }(P L F)=\frac{E P L-N P L}{N P L} \\
\text { SFI }=(-38.3 \times P L F)+(109.5 \times T S F)+(13.3 \times I T S F)-8.8 \\
\text { SSI }=[(108.44 \times T S F)+(31.85 \times I T S F)]-5.49
\end{gathered}
$$

\section{REFERENCES}

[1] X. Gu, F. Ding, Y. Yang, and J. Liu, "Construction of tissue engineered nerve grafts and their application in peripheral nerve regeneration," Prog. Neurobiol., vol. 93, no. 2, pp. 204-230, Feb. 2011.

[2] C. Radtke and P. M. Vogt, "Peripheral Nerve Regeneration : A Current Perspective," pp. 434-442, 2009.

[3] A. L. M. Gärtner, "Association of biomaterials and mesenchymal stem cells from Wharton's jelly of human umbilical cord for promotion of peripheral nerve regeneration: A functional and morphological study," 2013.

[4] R. Deumens, A. Bozkurt, M. F. Meek, M. a E. Marcus, E. a J. Joosten, J. Weis, and G. a. Brook, "Repairing injured peripheral nerves: Bridging the gap," Prog. Neurobiol., vol. 92, no. 3, pp. 245-276, Nov. 2010

[5] Y. Shirosaki, T. Okayama, K. Tsuru, S. Hayakawa, and A. Osaka, "Synthesis and cytocompatibility of porous chitosan-silicate hybrids for tissue engineering scaffold application," Chem. Eng. $J .$, vol. 137, no. 1, pp. 122-128, Mar. 2008.

[6] T. Pereira, A. Gartner, I. Amorim, P. Armada-da-Silva, R. Gomes, C. Pereira, D. M. Morais, M. L. França, M. A. Rodrigues, M. A. Lopes, J. D. Santos, A. L. Luís, and A. C. Maurício, "Biomaterials and stem cell therapies for injuries associated to skeletal muscular tissues," in Advances in Biomaterials Science and Biomedical Applications, 2013, pp. 1-26.

[7] A. C. Maurício, A. Gärtner, P. Armada-da-Silva, S. Amado, T. Pereira, A. P. Veloso, A. Varejão, A. L. Luís, and S. Geuna, "Cellular systems and biomaterials for nerve regeneration in neurotmesis injuries," Biomater. Appl. Nanomedicine, pp. 415440, 2011.

S. Amado, M. J. Simões, P. A. S. Armada da Silva, A. L. Luís, Y. Shirosaki, M. A. Lopes, J. D. Santos, F. Fregnan, G. Gambarotta, S. Raimondo, M. Fornaro, A. P. Veloso, A. S. P. Varejão, A. C. Maurício, and S. Geuna, "Use of hybrid chitosan membranes and N1E-115 cells for promoting nerve regeneration in an axonotmesis rat model," Biomaterials, vol. 29, no. 33, pp. 44094419, Nov. 2008.

[9] M. J. Simões, S. Amado, A. Gärtner, and P. A. S. Armada-dasilva, "Use of chitosan scaffolds for repairing rat sciatic nerve defects," Ital. J. Anat. Embryol., vol. 115, no. 3, pp. 190-210, 2010 .

[10] Y. Yuan, B. M. Chesnutt, G. Utturkar, W. O. Haggard, Y. Yang, J. L. Ong, and J. D. Bumgardner, "The effect of cross-linking of chitosan microspheres with genipin on protein release," Carbohydr. Polym., vol. 68, no. 3, pp. 561-567, Apr. 2007.

[11] Z. Osman and a. . Arof, "FTIR studies of chitosan acetate based polymer electrolytes," Electrochim. Acta, vol. 48, no. 8, pp. 993999, Apr. 2003.

A. L. Luís, J. M. Rodrigues, S. Geuna, S. Amado, Y. Shirosaki, J. M. Lee, F. Fregnan, M. A. Lopes, A. P. Veloso, A. J. Ferreira, J. D. Santos, P. A. S. Armada-da-Silva, A. S. P. Varejão, and A. C. Maurício, "Use of PLGA 90:10 Scaffolds Enriched with In VitroDifferentiated Neural Cells for Repairing Rat Sciatic Nerve Defects," Tissue Eng. Part A, vol. 14, no. 6, pp. 979-93, Jun. 2008.

[13] Y. Shirosaki, K. Tsuru, S. Hayakawa, A. Osaka, M. A. Lopes, J. D. Santos, and M. H. Fernandes, "In vitro cytocompatibility of MG63 cells on chitosan-organosiloxane hybrid membranes," Biomaterials, vol. 26, no. 5, pp. 485-93, Mar. 2005.

[14] Y.-L. Liu, Y.-H. Su, and J.-Y. Lai, "In situ crosslinking of chitosan and formation of chitosan-silica hybrid membranes with using $\gamma$-glycidoxypropyltrimethoxysilane as a crosslinking agent," Polymer (Guildf)., vol. 45, no. 20, pp. 6831-6837, Sep. 2004.

[15] J. M. Kerns, B. Braverman, A. Mathew, C. Lucchinetti, and A. D. Ivankovich, "A comparison of cryoprobe and crush lesions in the rat sciatic nerve.," Pain, vol. 47, no. 1, pp. 31-9, Oct. 1991.

[16] R. Koka and T. a Hadlock, "Quantification of functional recovery following rat sciatic nerve transection," Exp. Neurol., vol. 168, no. 1, pp. 192-5, Mar. 2001. 